\title{
La fiscalité des cultes en Europe : vers la fin de la singularité religieuse?
}

\section{Françoise Curtit}

\section{(2) OpenEdition}

\section{Journals}

Édition électronique

URL : http://journals.openedition.org/rdr/1032

DOI : $10.4000 /$ rdr.1032

ISSN : 2534-7462

Éditeur

Presses universitaires de Strasbourg

Édition imprimée

Date de publication : 10 mai 2016

Pagination : $39-54$

ISBN : 978-2-86820-954-2

ISSN : 2493-8637

\section{Référence électronique}

Françoise Curtit, «La fiscalité des cultes en Europe : vers la fin de la singularité religieuse ? », Revue du droit des religions [En ligne], 1 | 2016, mis en ligne le 12 février 2020, consulté le 19 novembre 2020. URL : http://journals.openedition.org/rdr/1032 ; DOI : https://doi.org/10.4000/rdr.1032

\section{(c) (7) (5)}

La revue du droit des religions est mise à disposition selon les termes de la Creative Commons Attribution - Pas d'Utilisation Commerciale 4.0 International - CC BY-NC 4.0. 


\section{LA FISCALITÉ DES CULTES EN EUROPE : VERS LA FIN DE LA SINGULARITÉ RELIGIEUSE ?}

\section{Françoise CURTIT}

Université de Strasbourg / CNRS, Droit, Religion, Entreprise et Société (DRES)

\section{RésuMÉ}

L'analyse des incitations fiscales dont bénéficient les organisations religieuses dans divers États européens permet de mettre en évidence leur alignement en la matière avec les organismes à but non lucratif et d'intérêt général. L'examen des mesures de déductibilité partielle des dons versés par les individus et les sociétés nous conduit à dégager un faisceau de critères suggérant l'application de mesures fiscales identiques pour toutes les entités développant des buts et activités d'intérêt général. Tout en permettant la suppression de mesures dérogatoires sources d'inégalité, l'accession à un statut de droit commun d'organisme d'intérêt général ouverte à l'ensemble des organisations religieuses et groupes de croyance, quels que soient leur statut et leur degré de reconnaissance par l'État, constituerait une approche renouvelée du financement indirect des cultes et un facteur de pluralisme confessionnel.

\section{ABSTRACT}

The analysis of tax incentives provided to religious organizations in European countries allows to highlight their alignment with non-profit organizations acting for public benefit. The examination of tax deductibility for donations from individuals and companies leads to identify common key criteria and to suggest the application of similar tax measures for all public benefit entities. While removing specific measures as sources of inequality, the assimilation of denominational organizations - whatever their legal status and degree of State recognition - into the category of public benefit associations would constitute an alternative approach to funding of religions contributing to religious pluralism. 
ans les pays européens, le mode de financement public des cultes est étroitement lié au statut juridique conféré aux organisations religieuses, lui-même issu d'un contexte historique et culturel particulier à chacun d'eux ${ }^{1}$. Le soutien financier de l'État est généralement accordé de façon sélective à l'Église majoritaire à laquelle est reconnu un rôle prépondérant (Église du Danemark, Église d'Angleterre, Église catholique en Italie ou en Espagne...) et aux confessions qui bénéficient de systèmes formalisés de coopération avec les pouvoirs publics (confessions reconnues ou enregistrées ou qui ont conclu un accord avec l'État en Belgique, Espagne, Italie, sociétés de droit public en Allemagne...). Les confessions religieuses peuvent par ailleurs être organisées principalement en référence au droit commun des associations - aux Pays-Bas notamment ou en France où les associations cultuelles et diocésaines sont issues d'une adaptation de la loi de 1901 sur la liberté d'association - ce qui n'exclut pas que des traitements préférentiels leur soient appliqués en matière de subventions et de fiscalité.

Nonobstant les différents modèles de relations État-religions, tous les États européens sont confrontés à des interrogations, voire à des remises en cause relatives aux mécanismes de financement des cultes, dans un contexte actuel caractérisé à la fois par la pluralisation religieuse, la sécularisation de la société et une situation de crise économique et financière. Il paraît alors opportun de réfléchir à un système de financement public des cultes qui soit d'une part plus adapté à la diversité religieuse et qui, sans abolir brutalement les méthodes actuelles de financement, favorise des mécanismes de soutien élaborés sur la base de critères d'égalité, de neutralité et de transparence. À cet égard, on soulignera qu'en sus des subventions directes, les États financent indirectement les confessions religieuses en leur octroyant des avantages fiscaux pour leurs organisations caritatives, éducatives, culturelles ou sociales, mais aussi pour leurs organisations à objet cultuel, en les alignant en la matière assez largement sur les associations de droit commun. Aussi allons-nous tenter d'explorer la politique fiscale comme un moyen de concevoir une nouvelle approche du financement public des cultes qui puisse s'appliquer au plus grand nombre possible d'organisations religieuses, en mettant l'accent sur leur alignement avec les organismes à but non lucratif et d'intérêt général (1). Nous prendrons l'exemple du régime des réductions d'impôts pour les dons alloués aux organisations religieuses pour préconiser des critères d'accès aux

1. Cet article est issu en partie de travaux menés dans le cadre du projet de recherche RELIGARE - Religious diversity and secular models in Europe financé par le $7^{\mathrm{e}}$ programmecadre de la Commission européenne (2010-2013). 
avantages fiscaux communs à l'ensemble des entités juridiques sur la base des éléments caractéristiques de l'intérêt général (2).

\section{MESURES FISCALES APPLICABLES AUX ORGANISATIONS RELIGIEUSES : DES ORGANISATIONS COMME LES AUTRES ?}

À côté de régimes fiscaux spécifiques mis en œuvre dans certains pays, la plupart des organisations religieuses bénéficient dans les États européens des déductions et exonérations accordées aux organisations à but non lucratif (1.1). En la matière, les incitations fiscales aux dons constituent une source non négligeable du soutien de l'État aux activités des organismes d'intérêt général, auxquels l'ensemble des organisations religieuses ne sont cependant pas assimilées (1.2).

\subsection{DES AVANTAGES FISCAUX PAR ASSIMILATION AUX ORGANISATIONS NON LUCRATIVES}

Certaines législations nationales prévoient la collecte d'un « impôt d'Église » pour les religions organisées dans le cadre du droit public, lié à l'appartenance religieuse du contribuable et calculé sur la base de l'impôt sur le revenu (Allemagne) ou de taxes fiscales locales (Danemark). Le paiement de l'impôt cesse lorsque le contribuable décide de quitter la confession religieuse en suivant une procédure officielle de retrait. Dans d'autres pays, les particuliers ont la possibilité d'allouer une partie de leur impôt sur le revenu à des confessions religieuses qui ont conclu un accord avec l'État, indépendamment de toute considération liée à leur appartenance religieuse (en Espagne, 0,7\% en faveur de l'Église catholique ou de besoins sociaux déterminés par le gouvernement ; en Italie, 0,8\% pour des actions humanitaires menées par l'État ou pour un culte choisi parmi l'Église catholique et les confessions avec entente). Ces dispositifs conçus spécifiquement pour des organisations religieuses sont étroitement inscrits dans un contexte national particulier.

Une autre catégorie de mesures fiscales, mise en œuvre de façon comparable dans l'ensemble des pays européens, consiste en une vaste gamme d'exonérations et déductions au profit de diverses organisations, y compris les organisations confessionnelles, entendues ici comme incluant les entités d'administration des cultes tout comme les organismes caritatifs, éducatifs, culturels ou d'action sociale gérés par ceux-ci. Certains de ces avantages sont calculés 
sur la base des activités et services dispensés (réduction ou exonération de la TVA sur la fourniture de biens et services, exonération ou taux réduit de la taxe professionnelle et de l'impôt sur les sociétés...), tandis que d'autres s'appliquent aux biens des organisations (exonération de droits de succession, exonération des droits de mutation à titre gratuit pour les dons et legs, exonération de l'impôt foncier, déductibilité fiscale pour les donateurs...). En ce qui concerne les organisations confessionnelles, les taxes relatives aux activités et services s'appliquent à leurs activités commerciales ou lucratives distinctes des finalités liées au culte. Ainsi, si certaines de leurs missions sont considérées comme servant des fins économiques (en matière de santé, de logement...), elles sont en général soumises aux mêmes règles fiscales que les entreprises. A contrario, les organisations religieuses peuvent être exemptées de la TVA sur l'acquisition et la construction d'immeubles ou sur la fourniture de biens et services, s'ils sont utilisés pour les besoins du culte ${ }^{2}$. Concrètement, une distinction doit être établie entre leurs activités commerciales et celles à but non lucratif, ou entre leurs objectifs cultuels et non cultuels. Au Royaume-Uni, par exemple, la construction ou l'acquisition d'un bâtiment est exonérée de la TVA si celui-ci est entièrement utilisé à des fins de bienfaisance. En pratique, une utilisation de $5 \%$ de la superficie à d'autres fins est cependant autorisée ${ }^{3}$. La règle paraît simple, mais n'est pas toujours facile à appliquer, puisqu'il s'agit d'identifier les surfaces attribuées respectivement à des activités commerciales et à des activités à but non lucratif ou à finalité cultuelle menées dans un même bâtiment. En matière d'impôts sur les biens immobiliers, les édifices affectés à l'exercice du culte bénéficient également d'exonérations ${ }^{4}$. En cas d'une utilisation mixte, à la fois commerciale et sans but lucratif, d'un même édifice, l'exonération s'applique si les locaux sont utilisés à titre principal pour le culte public (au moins 70\% de la superficie aux Pays-Bas ${ }^{5}$ ) ou est strictement limitée à la fraction de la propriété dans laquelle ont lieu les activités non commerciales (Imposta municipale unica, IMU en Italie ${ }^{6}$ ). Diverses exonérations

2. V. par ex., Belgique : Code de la TVA, art. 44, $211^{\circ}$; France : Code général des impôts, art. 261, $49^{\circ}$.

3. CRAnmer F., « Paying the piper? Public funding and supervision of religion in a secularized society (United Kingdom) », in Messner F., Public funding of religions in Europe, Farnham, Ashgate, 2015, p. 184.

4. V. par ex., Belgique: Code des impôts sur le revenu, art. 12 ; Angleterre et Pays de Galles : Places of Worship Registration Act 1855 ; France: Code général des impôts, art. 1382, 4 e) et art. 1407.

5. Cour suprême (Hoge Raad), 4 déc. 1991, n² 27.661.

6. Decreto-legge 24 gennaio 2012, n. 1, coordinato con la legge di conversione 24 marzo 2012, n. 27, Disposizioni urgenti per la concorrenza, lo sviluppo delle infrastrutture e la competitivita, art. 91-bis. 
de l'impôt foncier peuvent par ailleurs être accordées aux édifices religieux dans les mêmes conditions qu'aux autres établissements ouverts au public (taxe d'habitation en France ${ }^{7}$ ), qu'à toute personne morale de droit public (Grundsteuer en Allemagne $^{8}$ ) ou qu'aux organismes de bienfaisance ou à but non lucratif (Italie, Royaume-Uni).

Tout en maintenant la plupart du temps des mécanismes de financement direct pour les religions historiques, les États européens accordent ainsi à diverses organisations confessionnelles des avantages fiscaux en matière d'imposition des biens et des services similaires à ceux dont bénéficient les associations sans but lucratif. Elles ne se voient pas attribuer cependant de prérogatives particulières pour leurs activités économiques ou commerciales, et les États qui maintenaient des privilèges fiscaux en la matière ont dû les abroger sous la pression de la Commission européenne qui estime que les organisations religieuses sont des acteurs économiques comme les autres, et ne peuvent bénéficier de règles spécifiques qui entraîneraient une distorsion de concurrence, y compris dans le cadre de leurs activités caritatives ou sociales ${ }^{9}$. Leur assimilation au secteur non lucratif d'une part, et la logique de marché d'autre part, conduisent à une «banalisation» des organisations religieuses qui, en matière fiscale, se voient appliquer des règles de droit commun et sont considérées comme des organisations parmi d'autres.

En matière d'imposition des biens, le régime fiscal des dons et legs en vigueur dans les États européens est par ailleurs une illustration de l'alignement de certaines organisations confessionnelles sur les organisations à but non lucratif d'intérêt général.

\section{Code général des impôts, art. 1407.}

8. Grundsteuergesetz, $\S 3,3$ a).

9. V. en particulier les procédures formelles d'infraction lancées par la Commission européenne à l'Espagne, à l'Italie et au Danemark à propos d'avantages fiscaux accordés aux organisations religieuses: Press release IP/05/1620 of 16 December 2005, VAT Commission asks Spain to amend treatment of supplies of goods made to the Catholic Church ; Press release IP/12/1412 of 19 December 2012, State aid: Commission finds Italian ICI real estate tax exemptions for non-commercial entities incompatible and clears amended exemptions under new IMU law; Press release IP10/1319 of 12 October 2010, State aid: Commission opens probe into preferential real estate tax regime for non commercial entities in Italy; Press release IP/10/90 of 28 January 2010, VAT - Commission pursues infringement proceedings against Denmark regarding VAT exemptions. - V. CurTit F. and FORNEROD A., "State support for religions: European regulation », in Messner F., Public funding of religions in Europe, Farnham, Ashgate, 2015, p. 3-22. 


\subsection{DES INCITATIONS FISCALES AUX DONS PAR ASSIMILATION PARTIELLE AUX ORGANISMES D'INTÉRÊT GÉNÉRAL}

Les divers États européens développent des mécanismes d'incitation fiscale qui constituent une forme de soutien à un certain nombre de secteurs et d'activités, et c'est ainsi qu'ils favorisent notamment, dans le cadre de l'encouragement au mécénat, les dons au bénéfice des organismes à but non lucratif et d'intérêt général et, par assimilation, des organisations confessionnelles, en permettant aux contribuables de déduire de leurs impôts une partie des sommes versées, qu'il s'agisse de particuliers ou de sociétés.

Au Danemark, les organismes qui mènent des activités d'intérêt général sont ainsi autorisés à recevoir un financement privé qui implique une déduction de l'impôt sur le revenu, limité pour un particulier à 14500 couronnes danoises par $a^{10}$. Ce droit à déduction s'applique également aux dons versés aux communautés religieuses et plus d'une centaine d'entre elles bénéficient de ce mécanisme. En France, les dons à des associations d'utilité publique et à certains organismes d'intérêt général ouvrent droit pour les particuliers à une réduction de l'impôt sur le revenu égale à $66 \%$ des versements, dans la limite de $20 \%$ du revenu imposable ${ }^{11}$ et à une réduction de $60 \%$ des versements dans la limite de 0,5\% du chiffre d'affaires pour les entreprises assujetties à l'impôt sur le revenu ou à l'impôt sur les sociétés ${ }^{12}$. Les associations cultuelles bénéficient de ces incitations fiscales et font l'objet d'une mention explicite au sein des dispositions concernées ${ }^{13}$. La possibilité de bénéficier de divers avantages fiscaux constitue d'ailleurs l'enjeu principal de l'accès des organisations religieuses au statut d'association cultuelle accordé par les autorités préfectorales et fiscales à l'occasion de la déclaration de libéralités. En Allemagne, les successions et les dons sont déductibles dans la limite de 20\% du revenu imposable pour les particuliers ou $0,4 \%$ du chiffre d'affaires et des salaires et traitements annuels pour les sociétés ${ }^{14}$, à destination des organismes qui poursuivent exclusivement et directement des objectifs d'intérêt général, de bienfaisance ou religieux, lesquels incluent notamment la promotion de la religion (Förderung der Religion) et la réalisation de buts religieux (kirchliche Zwecke) conçue comme une activité de soutien à une société religieuse de droit

10. Loi sur l'évaluation de l'impôt (Ligningsloven, LBK nr 1041 af 15/09/2014), art. 8A.

11. Code général des impôts, art. 200

12. Code général des impôts, art. 238 bis

13. Code général des impôts, art. 200, 1 e) et art. 238 bis, 1. b)

14. Loi sur l'impôt sur le revenu (Einkommensteuergesetz, EStG), §10b) 
public $^{15}$. En Italie, les dons versés sous forme monétaire ou en nature par des personnes physiques ou par des entités soumises à l'impôt sur les sociétés sont déductibles à hauteur de 10\% du revenu déclaré des donateurs, et ce jusqu'à un maximum de 70000 euros par an lorsqu'ils bénéficient à des organisations à but non lucratif d'utilité sociale (organizzazioni non lucrative di utilità sociale, ONLUS) ${ }^{16}$, parmi lesquelles figurent explicitement les organisations confessionnelles avec lesquelles l'État a signé des ententes ou des accords ${ }^{17}$. Aux Pays-Bas, les confessions religieuses bénéficient d'avantages fiscaux grâce à leur statut d'organismes d'intérêt général (Algemeen Nut Beogende Instelling, $A N B I$ ), support juridique utilisé par ailleurs par un grand nombre d'organisations caritatives. Les dons à une ANBI sont déductibles de l'impôt sur le revenu ou sur les sociétés et l'organisme bénéficiaire est exempté de taxes sur ces sommes lorsqu'elles sont employées à des actions d'intérêt général ${ }^{18}$. $\mathrm{Au}$ Royaume-Uni, les communautés religieuses sont également destinataires d'avantages fiscaux en lien avec leur statut d'organismes de bienfaisance (charities). Les dons et legs effectués au bénéfice des charities peuvent faire l'objet d'un remboursement partiel au contribuable en fonction de son taux d'imposition. Ils permettent également aux organismes concernés de se voir reverser par l'État un complément représentant 20\% du montant du don avant imposition ${ }^{19}$. En Belgique enfin, les dons effectués auprès d'organisations agréées ${ }^{20}$ peuvent donner droit à une réduction d'impôt de $45 \%$ de leur montant. Les établissements publics de gestion des cultes (fabriques d'église par ex.) ne sont pas éligibles à ces mesures, toutefois les associations sans but lucratif qui leur sont liées en bénéficient ${ }^{21}$.

Les États européens appliquent donc des dispositions fiscales largement similaires visant à encourager les dons effectués en faveur des organismes sans but lucratif et d'intérêt général. Lorsque les organisations religieuses ne

15. Code des impôts (Abgabenordnung, AO), §51, §52, 2 et $\$ 54$.

16. Decreto-legge14 marzo 2005, n. 35, Disposizioni urgenti nell'ambito del Piano di azione per lo sviluppo economico, sociale e territoriale, art. 14.

17. Decreto legislativo 4 dicembre 1997, n. 460, Riordino della disciplina tributaria degli enti non commerciali e delle organizzazioni non lucrative di utilita' sociale, art. $10 \S 9$.

18. V. le site du service des impôts néerlandais : http://www.belastingdienst.nl [consulté le 12 févr. 2016].

19. Programme Gift Aid.

20. Organisations désignées expressément dans la loi et organisations agréées par le ministre des Finances ou par arrêté royal.

21. Sepulchre V., « Le financement des cultes et de la laïcité : aspects fiscaux », in Husson J.-F. (éd.), Le financement des cultes et de la laïcité : comparaison internationale et perspectives, Éd. namuroises, 2005, p. 215-241. 
disposent pas de facto d'un tel statut de droit commun, elles y sont assimilées en faisant l'objet d'une mention expresse au sein des dispositions fiscales. Dans plusieurs États cependant, seules les confessions religieuses majoritaires ou historiques, ou ayant noué des rapports institutionnels avec l'État, sont autorisées à bénéficier des avantages fiscaux liés aux dons ou au mécénat. C'est le cas notamment en Espagne, où seuls les dons à destination de l'Église catholique ou de l'un des trois groupes confessionnels ayant conclu un accord avec l'État permettent une déduction fiscale de l'impôt sur le revenu des particuliers ( $25 \%$ du montant du don) ou de l'impôt sur les sociétés (35\%)22. Ces déductions sont accordées pour les versements effectués au profit d'une large gamme d'entités à but non lucratif sur la base de leur rôle social et de leurs activités d'intérêt général ${ }^{23}$. Dans le cas espagnol, si l'extension de ces avantages aux organisations confessionnelles se fonde sur des critères similaires, c'est-à-dire sur leurs objectifs non lucratifs et leurs activités sociales et caritatives, il ne semble pas y avoir de raison objective de les limiter à l'Église catholique et aux confessions avec accords, sous peine de considérer qu'il s'agit là d'un traitement discriminatoire.

Le régime de déduction fiscale pour les dons constitue une source non négligeable de financement public aux activités - cultuelles et/ou caritatives selon les cas - des organisations confessionnelles institutionnellement reconnues par l'État. Tout en étant souvent spécifiquement désignées par les textes, celles-ci bénéficient de dispositions qui s'appliquent plus largement à un ensemble d'entités définies par leurs finalités non lucratives et d'intérêt général. Une intégration plus complète des organisations religieuses au droit commun des associations favoriserait une plus grande transparence et une meilleure clarté juridique, tout en permettant l'application de ces mesures fiscales à un maximum de confessions.

22. Ley 49/2002, de 23 de diciembre, de régimen fiscal de las entidades sin fines lucrativos y de los incentivos fiscales al mecenazgo.

23. Ibid., art. 1 à 3. 


\section{UN ACCÈS AUX AVANTAGES FISCAUX SUR LA BASE DE L'INTÉRÊT GÉNÉRAL}

En raison de leur ancrage culturel et de leur position institutionnelle, les religions historiques bénéficient généralement d'une présomption d'intérêt général qui leur permet de bénéficier d'emblée des avantages fiscaux afférents (2.1). Les religions implantées plus récemment sont, de façon implicite ou explicite, soumises au respect d'un certain nombre de critères auxquels elles doivent se conformer pour être enregistrées ou conclure des accords avec l'État (enracinement historique, nombre de membres, adhésion à des valeurs communes...). Ces exigences relatives à la pérennité de l'organisation, à son audience et à ses finalités peuvent être rapprochées des critères requis par les législations nationales pour l'obtention par les associations de droit commun d'un statut d'intérêt général leur permettant d'obtenir des avantages fiscaux. Ces critères appliqués à l'ensemble des entités juridiques constitueraient un facteur d'égalité entre les groupes religieux quel que soit leur statut et leurs liens institutionnels avec l'État (2.2).

\subsection{UNE PRÉSOMPTION D'INTÉRÊT GÉNÉRAL POUR LES ORGANISATIONS RELIGIEUSES BÉNÉFICIAIRES D'AVANTAGES FISCAUX}

Figurant explicitement parmi les bénéficiaires des dons ouvrant droit à une réduction d'impôt pour les donateurs, les associations cultuelles sont en France alignées en la matière sur les associations d'utilité publique, statut que toute association peut obtenir à l'issue d'une période probatoire de trois ans sur la base d'un faisceau de critères déterminés par la pratique administrative, incluant notamment un objet statutaire présentant un caractère d'intérêt général, un rayonnement dépassant un simple cadre local, un nombre minimum d'adhérents fixé à 200, un minimum annuel de ressources et des statuts garantissant l'existence de règles de fonctionnement démocratique et de transparence financière ${ }^{24}$. Le Conseil d'État avait souligné dans un avis du 15 mai 1962 que « les ouvres ou organismes tels que les

24. V. notamment question parlementaire de J.-Claude Flory n 16885 : JOAN Q 15 juill. 2008, p. 6174. - V. également les critères formant le «tronc commun d'agrément » pour les associations prévu par l'art. 25-1 de la loi n 2000-321 du 12 avril 2000 relative aux droits des citoyens dans leurs relations avec les administrations : $1^{\circ}$ Répondre à un objet d'intérêt général ; $2^{\circ}$ Présenter un mode de fonctionnement démocratique ; $3^{\circ}$ Respecter des règles de nature à garantir la transparence financière. Ces critères sont détaillés dans l'annexe 5 de la circulaire du Premier ministre du 18 janvier 2010 (NOR : PRMX1001610C). 
associations cultuelles, les associations diocésaines et diverses sociétés civiles, en répondant à un intérêt général, peuvent présenter nettement en raison des activités qu'ils exercent un caractère philanthropique, éducatif ou social ; [ils] rentrent ainsi dans le champ d'application de l'article 238 bis [du Code général des impôts]» ${ }^{25}$. Aux Pays-Bas, une ANBI doit remplir deux catégories de conditions, régissant d'une part ses objectifs et activités (au moins 90\% de ses activités doivent contribuer à l'intérêt général) et, d'autre part, la structure de l'organisation et sa comptabilité financière ${ }^{26}$. En Angleterre et au Pays de Galles, le Charities Act 2011 définit les charities comme des institutions établies uniquement à des fins de bienfaisance (for charitable purposes only) ${ }^{27}$, c'est-à-dire visant l'intérêt général (public benefit) ${ }^{28}$ : l'intérêt doit être identifiable et doit bénéficier au public en général ou à une partie suffisante du public ${ }^{29}$. Les fins de bienfaisance comprennent, entre autres, «l'avancement de la religion " (advancement of religion) et « la promotion des droits de l'homme, la résolution ou la réconciliation des conflits ou la promotion de l'harmonie religieuse ou raciale ou de l'égalité et de la diversité ${ }^{30}$. Selon la jurisprudence, l'avancement de la religion peut consister d'une part à rechercher de nouveaux adeptes ou adhérents et, d'autre part, à encourager et faciliter la pratique de la religion par les fidèles déjà présents, notamment par la fourniture et l'entretien d'un bâtiment utilisé pour la pratique religieuse, la conduite de cérémonies religieuses, la fourniture et l'entretien des vitraux et autres œuvres d'art dans les lieux de culte, ou encore la sensibilisation à la compréhension des croyances et pratiques religieuses, y compris par l'enseignement, l'assistance spirituelle et pastorale ${ }^{31}$. Les organismes de bienfaisance dont les objectifs comprennent l'avancement de la religion « ne sont pas tenus d'entreprendre des activités séculières en plus de leurs activités religieuses

25. CE, avis, 15 mai 1962, n 281012, cité in Messner F., Prélot P.-H., Woehrling J.-M. (dir.), Traité de droit français des religions, Paris, Litec, $2^{\mathrm{e}}$ éd. 2013, n 2343.

26. BeLAstingdienst, «Which conditions must be met by Public Benefit Organisations? » : http://www.belastingdienst.nl/wps/wcm/connect/bldcontenten/belastingdienst/business/ other_subjects/public_benefit_organisations/conditions_pbos/ [consulté le 12 févr. 2016].

27. Charities Act 2011, art. $1 \S 1$.

28. Charities Act 2011, art. 2 b). On a choisi de traduire «public benefit " par «intérêt général » pour éviter la confusion avec le mécanisme français de reconnaissance d'utilité publique.

29. The Charity Commission, Public benefit: rules for charities, 14 Feb. 2014 : https://www.gov. uk/public-benefit-rules-for-charities [consulté le 12 févr. 2016].

30. Charities Act 2011, art. $3 \S 1$ c) et h) [trad. par nous].

31. The Charity Commission, The advancement of religion for the public benefit, Dec. 2011 [trad. par nous] : https://www.gov.uk/government/uploads/system/uploads/attachment_data/ file/358531/advancement-of-religion-for-the-public-benefit.pdf [consulté le 12 févr. 2016]. 
dans le but de répondre à l'exigence d'intérêt général ${ }^{32}$. L'intérêt général est ainsi attribué d'emblée aux organisations confessionnelles, aussi bien à leurs structures d'organisation du culte qu'à leurs organismes caritatifs ou sociaux. Au Danemark, « tout but qui est généralement accepté par la population comme d'intérêt général ou de bienfaisance est qualifié d'intérêt général en vertu de l'article $8 \mathrm{~A}$ de la loi sur l'évaluation de l'impôt $»^{33}$. Pour être reconnu d'intérêt général, le but poursuivi doit en outre concerner « des bénéficiaires potentiels qui ne peuvent pas être limités, géographiquement ou d'une autre manière, à un groupe de moins de 40000 personnes ${ }^{34}$. Des exigences de transparence et de bonne gouvernance et l'obligation de rendre des comptes sont également requises des organismes concernés. Par ailleurs, « les sociétés ou entités de nature religieuse peuvent être considérées comme d'intérêt général en vertu de l'article $8 \mathrm{~A} »{ }^{35}$. En Espagne, pour bénéficier des avantages fiscaux définis dans la loi 49/2002 36 , les organismes d'intérêt général doivent poursuivre des objectifs d'intérêt public et ne peuvent pas exercer des activités économiques hors de la finalité sociale de l'entité. Parmi d'autres exigences, ils doivent se conformer à des règles comptables spécifiques et présenter un rapport financier annuel détaillant leurs revenus et dépenses par catégories et projets ${ }^{37}$. En Italie, les organisations à but non lucratif d'utilité sociale (ONLUS) sont des organismes à caractère privé, avec ou sans personnalité juridique, dont les statuts prévoient la poursuite exclusive de fins de solidarité sociale $^{38}$. Ils ont pour obligation de rédiger un bilan financier annuel. En Allemagne, un organisme poursuit des finalités d'intérêt général si ses activités visent à aider de manière désintéressée la collectivité (die Allgemeinheit) dans le domaine matériel, spirituel ou moral. Cette aide ne doit pas s'adresser à un cercle de personnes, restreint par exemple aux membres d'une famille ou aux

32. Ibid. [trad. par nous].

33. Friss Hansen S. and Graff Nielsen J., Taxation of charities [Denmark], European Association of Tax Law Professors, Annual Meeting Rotterdam, 31 May - 2 June 2012, p. 3 [trad. par nous] : http://eatlp.org/index.php/documents/rotterdam-2012 [consulté le 12 févr. 2016].

34. Ibid., p. 4 [trad. par nous].

35. Ibid., p. 3 [trad. par nous].

36. Ley 49/2002, précit.

37. BÁez A. and Pedreira J., Taxation of charities in Spain, European Association of Tax Law Professors, Annual Meeting Rotterdam, 31 May - 2 June 2012 : http://eatlp.org/index.php/ documents/rotterdam-2012 [consulté le 12 févr. 2016].

38. Decreto legislativo 4 dicembre 1997, n. 460, Riordino della disciplina tributaria degli enti non commerciali e delle organizzazioni non lucrative di utilita' sociale, art. 10. 
employés d'une entreprise, ou durablement limité par des caractéristiques spatiales ou professionnelles ${ }^{39}$.

Dans l'ensemble des pays européens, les avantages fiscaux constituent un moyen pour l'État de favoriser le développement d'activités non lucratives à caractère éducatif, social, culturel, et aussi religieux. Pour pouvoir en bénéficier, les organisations doivent satisfaire des exigences relatives à leurs objectifs, mais aussi à l'étendue du public visé, à l'organisation de leur gouvernance et à la reddition de leurs comptes financiers, en plus de diverses procédures formelles parfois requises (contenu des statuts, déclaration ou enregistrement...). Lorsqu'elles ne revêtent pas la forme juridique de droit commun des associations d'intérêt général, les organisations confessionnelles leur sont assimilées, qu'elles soient explicitement citées parmi la liste des bénéficiaires des mesures fiscales ou pas. De façon générale, les religions, en tout cas celles qui bénéficient d'une procédure de "reconnaissance » de droit ou de fait par l'État, voient leurs activités gratifiées d'une présomption d'intérêt général. Leurs finalités religieuses (voir la mention de « l'avancement de la religion » parmi les fins de bienfaisance dans les législations allemande et britannique), et pas uniquement sociales ou caritatives, seraient susceptibles d'être bénéfiques à l'ensemble de la société dans la mesure où elles favoriseraient par exemple l'altruisme et l'intégration sociale. Ces organisations religieuses sont considérées au même titre que les associations qui développent une activité désintéressée pour le bien commun et sont soutenues financièrement en tant que telles.

Cette approche fiscale centrée sur les activités et les finalités poursuivies tend à banaliser les entités confessionnelles en les assimilant à des associations sans but lucratif et d'intérêt général, sans plus les distinguer par un statut ou des dispositions juridiques particuliers. Tout comme en matière d'impôts sur les biens immobiliers, un même régime fiscal s'applique en matière de déductibilité des dons à toute une série d'entités, parmi lesquelles les organisations religieuses sont des acteurs comme les autres. Mais si la notion d'intérêt général est centrale dans ce dispositif, il ne s'agit pas là d'une condition suffisante. Cet objectif doit bénéficier à un nombre suffisamment élevé de personnes et ne peut donc être limité à une communauté réduite. On peut estimer qu'en la matière des restrictions éventuelles posées par une organisation concernant le public visé par ses activités devraient être justifiées, raisonnables et objectives, sous peine d'être jugées discriminatoires et d'invalider son accès aux avantages fiscaux. Par ailleurs, la condition d'un but non lucratif, ou en

39. Code des impôts (Abgabenordnung, AO), §52(1). 
tout cas d'une activité non majoritairement à but lucratif, ne dispense pas l'organisme de devoir rendre des comptes en matière de gestion financière et de fonctionnement démocratique de ses instances. Sur ces deux points, les confessions religieuses bénéficient jusqu'à présent de contraintes relativement faibles eu égard au principe de respect de leur autonomie d'organisation et de fonctionnement, particulièrement en ce qui concerne leurs entités proprement cultuelles ${ }^{40}$.

\subsection{DÉFINIR DES CRITÈRES D'ACCÈS AUX AVANTAGES FISCAUX GARANTS DE L'ÉGALITÉ ENTRE GROUPES RELIGIEUX}

La politique fiscale promue par les États constitue une voie de financement non négligeable pour les organisations religieuses, financement direct par les donateurs et indirect par l'État pour qui les déductions octroyées constituent au final un manque à gagner. Dans la plupart des pays européens, les confessions religieuses bénéficient d'incitations fiscales pour leurs activités qui contribuent à l'intérêt général, mais celles-ci sont en général réservées aux confessions qui ont conclu un accord avec l'État (Espagne, Italie) ou ont acquis un statut juridique particulier (Allemagne, France). Dans quelques cas seulement (Danemark, Pays-Bas, Royaume-Uni), les avantages fiscaux sont accessibles à un grand nombre d'entités menant des activités d'intérêt général, y compris une grande variété d'organisations religieuses. Dans ce domaine comme dans d'autres, l'État est tenu de respecter le principe d'égalité entre les groupes religieux, toute différence de traitement devant pouvoir s'appuyer sur des justifications objectives et raisonnables. En matière fiscale, les différences entre les religions historiquement majoritaires et les religions minoritaires ou implantées plus récemment sont parfois disproportionnées. Ainsi en 1994, la Commission européenne des droits de l'homme notait qu'en droit français, le choix de doter les associations cultuelles d'un statut juridique plus favorable s'explique par des considérations historiques, mais que néanmoins « le Gouvernement n'a pas fourni de justification à la différence de traitement opérée par la législation française en matière de libéralités entre les associations cultuelles d'une part et les autres associations d'autre part. La Commission n'aperçoit, quant à elle, aucune justification objective et

40. Autonomie des cultes reconnue par la CourEDH comme indispensable au pluralisme dans une société démocratique et donc au cœur de la protection offerte par l'article 9 de la Convention. - V. en particulier CEDH, Gde ch., 26 oct. 2000, n 30985/96, Hassan et Tchaouch c/ Bulgarie, $\$ 62$ ou CEDH, Gde ch., 9 juill. 2007, n² 2330/09, Sindicatul « Păstorul cel Bun » c/ Roumanie, $\$ 136$ et s. 
raisonnable de maintenir un système qui défavorise à un tel degré les associations non cultuelles ${ }^{41}$.

Afin de permettre aux groupes religieux minoritaires d'être mieux soutenus financièrement, les États pourraient intégrer toutes les organisations religieuses dans les mécanismes d'exemptions ou de réductions fiscales accordées aux organismes d'intérêt général, dès lors qu'elles remplissent les conditions requises pour accéder à ce statut. Les avantages fiscaux seraient ainsi ouverts aux confessions indépendamment de leur doctrine, de leur rôle historique et de leur statut juridique et sur la seule base de leurs objectifs et activités, conditions non discriminatoires favorisant l'égalité entre les groupes religieux et garantissant ainsi la liberté de religion et la neutralité de l'État.

Les déductions fiscales pour les dons constituent un point clé de la politique de soutien des organisations religieuses, en ce qu'elles favorisent leur financement par leurs membres et adeptes, et au-delà par tout particulier ou personne morale qui souhaite participer à leur développement. Les types de relations nouées avec l'État ne constituent plus ici l'élément déterminant pour désigner les confessions bénéficiaires, les contribuables sont encouragés à faire des versements aux communautés religieuses de leur choix, celles-ci étant alors financées relativement à leur audience et leur attractivité, et donc de façon d'autant plus importante qu'elles promeuvent des valeurs partagées par le plus grand nombre. La recrudescence des « sorties d'Églises » ces dernières années en Allemagne, motivées en partie par un refus de payer l'impôt ecclésiastique, illustre la nécessité de s'orienter vers un modèle de financement qui privilégie et encourage les dons volontaires, respectueux de la liberté individuelle de religion conçue comme la liberté de croire ou de ne pas croire.

Dans l'objectif d'étendre les avantages fiscaux au plus grand nombre possible d'organisations religieuses, il revient aux pouvoirs publics de définir des critères d'accès qui soient communs à l'ensemble des entités juridiques et sur la seule base des éléments caractéristiques de l'intérêt général déjà évoqués (pérennité, audience et finalités de l'organisation). Le concept d'intérêt général étant étroitement lié aux attentes sociales, cette définition devra être régulièrement adaptée à l'évolution des besoins ${ }^{42}$, ce qui semble néanmoins plus aisé

41. Comm. EDH, Rapport 6 juillet 1994, n 14635/89, Union des athées c/ France, \$78 - V. dans ce numéro, Gonzalez G., « Le financement des cultes et la Convention européenne des droits de l'homme».

42. V. France. Conseil d’État, Réflexions sur l'intérêt général - Rapport public 1999, 30 nov. 1998 : «La plasticité est consubstantielle à l'idée d'intérêt général, qui peut ainsi évoluer en fonction des besoins sociaux à satisfaire et des nouveaux enjeux auxquels est confrontée la société. » : http://www.conseil-etat.fr/Decisions-Avis-Publications/Etudes-Publications/ 
que de déterminer ce qui est ou pas cultuel, ou ce qui contribue à l'avancement de la religion. Sans pouvoir exercer d'ingérence excessive, les autorités administratives et fiscales conserveraient par conséquent une certaine marge d'appréciation pour établir et vérifier les conditions d'accès aux avantages fiscaux, ce qui ne les dispense pas de devoir divulguer des informations claires et fiables concernant leurs critères d'appréciation, afin de les rendre aussi indiscutables que possible et de respecter pleinement le principe d'égalité de traitement. En retour, elles devraient pouvoir exiger des organisations bénéficiaires la tenue d'une comptabilité ainsi qu'une information régulière sur la provenance de leurs financements et sur la façon dont elles ont utilisé les dons ayant bénéficié d'une déductibilité, démarche par ailleurs conforme aux attentes légitimes des citoyens en matière de transparence financière. Ces objectifs d'égalité et de transparence s'accompagnent en outre d'une mission de clarification. Il convient en effet dans de nombreux cas de simplifier les législations nationales, en supprimant des dispositions fiscales propres aux cultes (ou à certains cultes) qui sont parfois difficilement lisibles et enfreignent le principe de sécurité juridique. La refonte des dispositions fiscales pourrait en outre être l'occasion d'accroître leur efficacité en améliorant l'attractivité des incitations aux dons, selon les cas en augmentant les taux de déductibilité, en élargissant les plafonds de déduction, en déduisant les dons de l'impôt dû plutôt que du revenu imposable, ou encore en instaurant un système d'avantages fiscaux qui varieraient en fonction du montant des dons.

À une époque où les mécanismes de financement public des cultes font l'objet dans nombre de pays européens de débats publics au sujet de leur montant et de leur légitimité, la voie des incitations fiscales apparaît comme un moyen de suppléer progressivement au financement direct de l'État, tout en assurant néanmoins un certain soutien aux organisations confessionnelles. L'exemple de la déductibilité partielle des dons des particuliers ou des sociétés nous permet de mettre en évidence un faisceau de critères caractérisant les organisations d'intérêt général pouvant bénéficier d'avantages fiscaux, lequel pourrait être appliqué à l'identique aux organisations confessionnelles quelles qu'elles soient, sans plus les distinguer par des mesures spécifiques. À l'exemple des législations néerlandaise et britannique qui ont déjà largement intégré les organisations religieuses dans le droit commun des organismes d'intérêt général, cette «banalisation » fiscale serait garant du respect des principes de neutralité et de transparence et un facteur de pluralisme confessionnel,

Rapports-Etudes/Reflexions-sur-l-interet-general-Rapport-public-1999 [consulté le 12 févr. 2016]. 
permettant une meilleure prise en compte du rôle et de l'influence actuels des différentes confessions indépendamment de leur position historique, culturelle ou institutionnelle. 